\title{
The Hartree-Fock ground state of the three-dimensional electron gas
}

\author{
Shiwei Zhang ${ }^{1}$ and D. M. Ceperley ${ }^{2}$ \\ ${ }^{1}$ Department of Physics, College of William and Mary, Williamsburg, VA 23187, USA \\ ${ }^{2}$ NCSA and Department of Physics, University of Illinois at Urbana-Champaign, Urbana, IL 61801, USA
}

In 1962, Overhauser showed that within Hartree-Fock (HF) the electron gas is unstable to a spin density wave (SDW) instability. Determining the true HF ground state has remained a challenge. Using numerical calculations for finite systems and analytic techniques, we study the HF ground state of the 3D electron gas. At high density, we find broken spin symmetry states with a nearly constant charge density. Unlike previously discussed spin wave states, the observed wave vector of the SDW is smaller than $2 k_{F}$. The broken-symmetry state originates from pairing instabilities at the Fermi surface, a model for which is proposed.

PACS numbers: 71.10.Ca,71.10.-w,71.15.-m,75.30.Fv

The three-dimensional electron gas is one of the basic models of many-body physics, and has been investigated for over 70 years [1, 2, 3, 4, 5, 6, 7]. As the simplest model system representing an itinerant metal, it consists of interacting electrons in a uniform neutralizing charge background, described by the Hamiltonian:

$$
H=-\frac{\hbar^{2}}{2 m} \sum_{i} \nabla_{i}^{2}+\frac{1}{2} \sum_{i \neq j} \frac{e^{2}}{\left|\mathbf{r}_{i}-\mathbf{r}_{j}\right|}+\text { constant }
$$

where the sums are over particle indices. Its properties are routinely used in density functional theory, e.g., in local density approximations, as a reference state in calculations of electronic structure of real materials [6].

The simplest approach to an interacting many-fermion system such as the electron gas (jellium) is the mean-field Hartree-Fock (HF) method, which finds the Slater determinant wave function minimizing the variational energy. In unpolarized jellium, the "conventional" solution is a paramagnetic state with spin symmetry, the restricted $\mathrm{HF}$ (rHF) solution in quantum chemistry.

The rHF solution, however, is not the exact HF ground state of jellium. In 1962, Overhauser [3] proved that the rHF solution is unstable with respect to spin and charge fluctuations at any density. The global minimum energy state within HF is a spontaneously broken symmetry state. The properties of this global ground state have remained unknown [7]. This is surprising, given the fundamental importance of both the electron gas and the HF approach. The correlation energy of the homogeneous electron gas is a commonly used fundamental concept, but its definition is in terms of the HF energy of the electron gas.

In this paper, we numerically find the HF ground state for finite systems. Our motivation, aside from solving this mathematical puzzle, was to understand the mechanism for the broken symmetry state. Further, it was hoped that the solution would suggest candidate ground states, for jellium or for other systems, that can then be explored by accurate many-body approaches such as quantum Monte Carlo [5, 8]. We focus on high and medium densities. An analytic approach is used to augment and extend the results to the thermodynamic limit. We find that a different pairing instability characterizes the high-density ground state.

We consider $N\left(N_{\uparrow}=N_{\downarrow}=N / 2\right)$ electrons in a cubic supercell of volume $\Omega=L^{3}$. The density is specified by the average distance between electrons: $r_{s} \equiv$ $(3 \Omega / 4 \pi N)^{1 / 3} / a_{B}$. We write a Slater determinant as

$$
|\Phi\rangle=\left|\phi_{1}^{\uparrow}, \phi_{2}^{\uparrow}, \cdots, \phi_{N_{\uparrow}}^{\uparrow}\right\rangle \otimes\left|\phi_{1}^{\downarrow}, \phi_{2}^{\downarrow}, \cdots, \phi_{N_{\downarrow}}^{\downarrow}\right\rangle,
$$

with $\left|\phi_{j}^{\sigma}\right\rangle=\sum_{\mathbf{k}} c_{j}^{\sigma}(\mathbf{k})|\mathbf{k}\rangle$ where $|\mathbf{k}\rangle$ is a plane-wave basis function. In the rHF solution, any $|\mathbf{k}\rangle$ with $k \equiv|\mathbf{k}| \leq k_{F}$ is fully occupied, while all others are empty. Our basis contains all plane-waves with $k<k_{\text {cut }}$.

To find the global ground state, i.e., the unrestricted HF (uHF) solution, we use an iterative projection

$$
\left|\Phi^{(m+1)}\right\rangle=e^{-\tau H_{\mathrm{HF}}\left(\Phi^{(m)}\right)}\left|\Phi^{(m)}\right\rangle,
$$

where $H_{\mathrm{HF}}\left(\Phi^{(m)}\right)$ is the HF Hamiltonian, i.e., the meanfield approximation of Eq. (11). The wave function remains a single Slater determinant in the projection [8]. If $\tau$ is sufficiently small, the energy will decrease in each step and the projection will converge to a HF solution as $m \rightarrow \infty$. To ensure that the solution is not a local minimum, we often start from multiple random initial states $\left|\Phi^{(0)}\right\rangle$ and verify that the same final state is reached.

The smallness of the energy scale relevant to the symmetry breaking at high density presents a difficulty; finite-size effects can easily be larger than differences in energy of different phases. As a consequence, the stable structures vary wildly with $N$. There are subtle commensuration effects in both $\mathbf{r}$-space (Wigner crystal) and k-space (spin waves). For example, in open-shell systems there is always a uHF solution, since a broken symmetry state can be formed in a partially filled shell to lower the exchange energy, with no cost to the kinetic or Hartree energy (see below). In closed-shell systems, on the other hand, there seems to be a critical value of $r_{s}^{c}(N)$, below which no uHF state exists for a given value of $N$ under periodic boundary condition (PBC). 
To break the shell structure, we impose twisted boundary conditions [9] on the orbitals (and hence on the wavefunction): $\phi(\mathbf{r}+L \hat{\alpha})=e^{i 2 \pi \theta_{\alpha}} \phi(\mathbf{r})$ where $\alpha=x, y, z$. This applies a shift of $\mathbf{k}_{\theta}=2 \pi \vec{\theta} / L$ to the plane-wave basis. $\vec{\theta}=0$ corresponds to $\mathrm{PBC}$, the $\Gamma$-point for solids. For generic twist angles $\vec{\theta}$, the rHF solution is nondegenerate.

Let us write the Hamiltonian in second quantized form, omitting an overall constant:

$$
\hat{H}=\frac{\hbar^{2}}{2 m} \sum_{\sigma, \mathbf{k}} \mathbf{k}^{2} c_{\mathbf{k}, \sigma}^{\dagger} c_{\mathbf{k}, \sigma}+\frac{1}{2 \Omega} \sum_{\Lambda} \frac{14 e^{2}}{\mathbf{Q}^{2}} \hat{V}(\Lambda),
$$

where $c_{\mathbf{k}, \sigma}^{\dagger}$ and $c_{\mathbf{k}, \sigma}$ are creation and annihilation operators. In the second term, $\Lambda$ denotes the variables $\left\{\mathbf{k}, \mathbf{k}^{\prime}, \mathbf{Q}, \sigma, \sigma^{\prime}\right\}, \mathbf{Q}$ is a reciprocal lattice vector, the ' on the summation indicates $\mathbf{Q} \neq 0$, and

$$
\hat{V}(\Lambda) \equiv c_{\mathbf{k}-Q, \sigma}^{\dagger} c_{\mathbf{k}^{\prime}+Q, \sigma^{\prime}}^{\dagger} c_{\mathbf{k}^{\prime}, \sigma^{\prime}} c_{\mathbf{k}, \sigma}
$$

The HF Hamiltonian $\hat{H}_{\mathrm{HF}}\left(\Phi^{(m)}\right)$ needed in Eq. (3) is the same as $\hat{H}$, but with $\hat{V}(\Lambda)$ replaced by the linearized form $\hat{V}_{\mathrm{HF}}(\Lambda)=\hat{v}(\Lambda)-\langle\hat{v}(\Lambda)\rangle / 2$, where

$$
\begin{aligned}
\hat{v}(\Lambda) \equiv & 2\left[\left\langle\sum_{\mathbf{k}^{\prime \prime}} c_{\mathbf{k}^{\prime \prime}, \sigma^{\prime}}^{\dagger} c_{\mathbf{k}^{\prime \prime}-\mathbf{Q}, \sigma^{\prime}}\right\rangle \delta_{\mathbf{k}^{\prime}, \mathbf{k}+\mathbf{Q}}\right. \\
& \left.-\left\langle c_{\mathbf{k}^{\prime}+\mathbf{Q}, \sigma^{\prime}}^{\dagger} c_{\mathbf{k}+\mathbf{Q}, \sigma^{\prime}}\right\rangle \delta_{\sigma, \sigma^{\prime}}\right] c_{\mathbf{k}, \sigma}^{\dagger} c_{\mathbf{k}^{\prime}, \sigma}
\end{aligned}
$$

The expectation $\langle.$.$\rangle is with respect to \left|\Phi^{(m)}\right\rangle$. The variational energy is $E_{v}(\Phi) \equiv\langle\Phi|\hat{H}| \Phi\rangle /\langle\Phi \mid \Phi\rangle$. We also compute a "growth estimator" of the energy, $E_{g} \equiv$ $-\ln \left[\left\langle\Phi^{(m+1)} \mid \Phi^{(m+1)}\right\rangle /\left\langle\Phi^{(m)} \mid \Phi^{(m)}\right\rangle\right] / 2 \tau$, in the projection. At convergence, $E_{v}\left(\Phi^{(m)}\right)=E_{v}\left(\Phi^{(m+1)}\right)=E_{g}$, which means that $\left|\Phi^{(m)}\right\rangle$ is an eigenfunction of $\hat{H}_{\mathrm{HF}}$. Hence, the projection gives a true solution of the $\mathrm{HF}$ Hamiltonian, not just a Slater determinant with a variational energy lower than the rHF value.

Calculations were carried out with a set of random $\vec{\theta}$ and the results averaged and errors estimated. For each $N$, a fixed set of $\mathbf{k}_{\theta}$-points were used at different values of $r_{s}$ to correlate the runs. At larger $r_{s}$, less statistical accuracy is needed and fewer $\mathbf{k}_{\theta}$-points were used. Typically the plane-wave basis cutoff was set to $k_{\text {cut }} \sim 2-3 k_{F}$, i.e., a kinetic energy cutoff of $5-10 E_{F}$. The resulting basis set error is negligible for all but the largest $r_{s}(=7)$. Fast Fourier transform (FFT) techniques were used to speed up each step in the projection [8], and the orbitals re-orthonormalized as necessary.

The top panel of Fig. 1 shows the energy difference $\delta E$ between the $\mathrm{uHF}$ ground state and the $\mathrm{rHF}$ state. As $r_{s}$ is reduced, the magnitude of $\delta E$ decreases before becoming nearly flat at high densities $\left(r_{s}<3\right)$ for each value of $N$. The energy lowering remains finite across $r_{s}$, showing that the broken symmetry uHF solution exists for all densities, consistent with Overhauser's proof [3]. At low $r_{s}, \delta E$ is roughly $-10^{-4} \mathrm{Ry}$ in the large-sized systems.

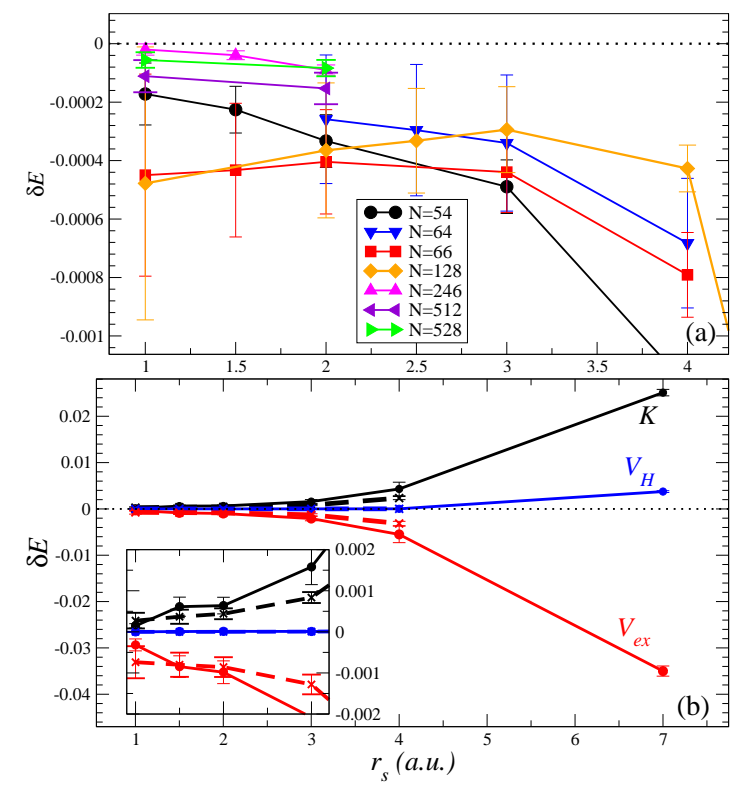

FIG. 1: (color online) Energy differences (in Ry) between the $\mathrm{HF}$ ground state and the rHF state. (a) The energy lowering per electron, $\delta E=\left(E-E_{\mathrm{rHF}}\right) / N$, vs. $r_{s}$ for different values of $\mathrm{N}$ [10]. (b) The three components of $\delta E$, kinetic $(K)$, Hartree $\left(V_{H}\right)$, and exchange $\left(V_{e x}\right)$, for $N=54$ (solid lines) and $N=$ 66 (dashed lines). The inset is an enlargement of the low $r_{s}$. Error bars are estimated from the results for various $\mathbf{k}_{\theta^{-}}$ points.

Calculations for larger systems are needed to clarify its behavior at small $r_{s}$. The energy differences are very small compared to the rHF energy which, at $N \rightarrow \infty$, is $E_{\mathrm{rHF}} / N=\left(2.21 / r_{s}^{2}-0.916 / r_{s}\right)$ Ry: the relative energy reduction vanishes as $r_{s} \rightarrow 0$. From the bottom panel, we see that the lower total energy of the uHF ground state is achieved by reducing the exchange energy at the cost of increased kinetic energy (exciting electrons into $|\mathbf{k}\rangle$ states with $k>k_{F}$ ). The Hartree energy remains unchanged up to $r_{s} \sim 3$.

We find that the momentum distribution in the $\mathrm{uHF}$ solution is spin-independent, i.e., $n_{\uparrow}(\mathbf{k})=n_{\downarrow}(\mathbf{k})$ is an unbroken symmetry in the broken symmetry ground state. This is illustrated in Fig. 2 for $r_{s}=3$, but holds in all our calculations when fully converged, at all $r_{s}$ and $N$.

Figure 2 also shows that, as $r_{s}$ decreases, the occupancy of $k>k_{F}$ states becomes less pronounced, and significant modifications to the Fermi sphere become increasingly confined to the immediate vicinity of the Fermi surface (FS). At small $r_{s}$, such modifications tend to be principally single pairing states. An example is seen at $r_{s}=1$ in $N=54$ : two plane-wave vectors are involved, such that a pair of rHF orbitals $\left|\phi^{\uparrow}\right\rangle=\left|\phi^{\downarrow}\right\rangle=|\mathbf{k}\rangle$ become

$$
\left|\phi^{\uparrow}\right\rangle=c_{\mathbf{k}}|\mathbf{k}\rangle \pm c_{\mathbf{k}^{\prime}}\left|\mathbf{k}^{\prime}\right\rangle
$$

where $k \leq k_{F}$ and $k^{\prime}>k_{F}$, and $\left|c_{\mathbf{k}}\right|^{2}+\left|c_{\mathbf{k}^{\prime}}\right|^{2}=1$. Such a pairing state by itself forms a linear spin-density wave 


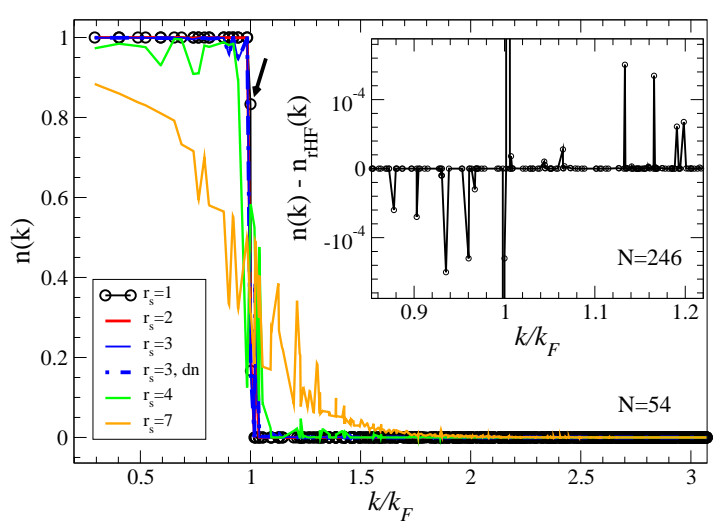

FIG. 2: (color online) Momentum distribution $n(\mathbf{k})$ at different $r_{s}$ vs. $k / k_{F}$. The main graph has $N=54$, with $\vec{\theta}=(-0.368,0.172,-0.364)$. The arrow indicates $k_{F}$. The inset shows $\left[n(\mathbf{k})-n_{\mathrm{rHF}}(\mathbf{k})\right]$ at $r_{s}=1$ for $N=246$, with $\vec{\theta}=(-0.494,-0.425,0.144)$. Note the primary spike at $k_{F}$, and the paired smaller spikes with one on each side of $k_{F}$.

(SDW), with constant charge density and unchanged Hartree energy. As the inset in Fig. 2 shows, additional pairing states form in the uHF solution involving wave vectors further from the FS. Although their amplitudes become very small as $r_{s}$ is reduced, these are important to the true uHF ground state, as we discuss later.

Real-space properties are examined in Fig. 3. The charge density is $\rho(\mathbf{r})=n_{\uparrow}(\mathbf{r})+n_{\downarrow}(\mathbf{r})$ and the spin density is $\sigma(\mathbf{r})=n_{\uparrow}(\mathbf{r})-n_{\downarrow}(\mathbf{r})$. We measure their Fourier transforms, e.g., $S_{\rho}(\mathbf{q})=\left|\int \rho(\mathbf{r}) e^{i \mathbf{q} \cdot \mathbf{r}} d \mathbf{r}\right|^{2} / N$. At $r_{s}=7$, the $N=54$ system is an antiferromagnetic bcc Wigner crystal. As $r_{s}$ decreases, electrons become less localized and less particle-like. Fluctuation in the charge density becomes much smaller, as indicated by the rapid reduction in $S_{\rho}(\mathbf{q})$. This is consistent with the vanishing Hartree energy in Fig. 1. Although $S_{\sigma}(\mathbf{q})$ also decreases with $r_{s}$, it is much larger and spin symmetry remains broken. At $r_{s}=4$, the SDW no longer has a bcc structure, and its symmetry between $x, y$, and $z$ is broken. At small $r_{s}$, the electrons are highly delocalized and wavelike. Charge variations are effectively compensated for by spatial "double occupancy" of $\uparrow$ and $\downarrow$ electrons, as in the pairing state discussed in Fig. 2 .

We measure the characteristic wave vector of the spin or charge density wave by $q=|\mathbf{q}|$, where $\mathbf{q}$ is the peak position of $S(\mathbf{q})$. A wave vector of $q_{\sigma} \sim 2 k_{F}$ seems to have always been assumed in previous investigations of the SDW states [3, 7]. However, we find the maximum spin ordering is at smaller wave vectors, as shown in Fig. 3 for $N=54$. Consistent results are seen for larger $N$, e.g. at $r_{s}=2, q_{\sigma} / k_{F}=1.2(2), 1.4(2), 1.5(3)$, and 1.5(2) for $N=66,128,246$, and 528 , respectively.

We next prove analytically that an SDW instability whose wave vector is $q_{\sigma}<2 k_{F}$ indeed exists. Let us con-

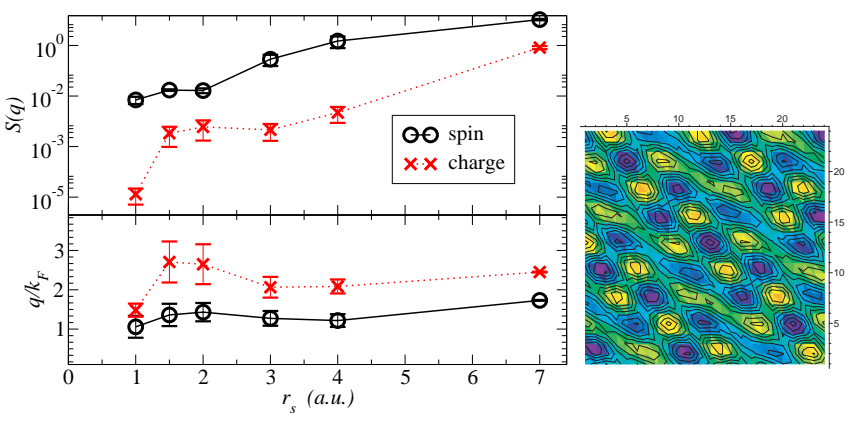

FIG. 3: (color online) Left: Peak values and locations of the Fourier transforms of spin and charge densities for different values of $r_{s}$ in $N=54$. Errors are estimated from the values at different $\mathbf{k}_{\theta}$-points. Note the logarithmic scale in $S(q)$. Right: Contour plot of the spin density $\sigma(x, y, z)$ for a slice parallel to the $x-y$ plane. The system has $N=512$ and $r_{s}=1$. The q-vector $[2 \overline{6} \overline{3}]$ has the leading $S_{\sigma}(\mathbf{q})$ value, followed by

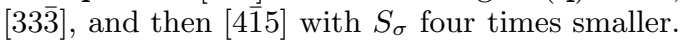

sider a system of large but finite $N$, with $\mathbf{k}_{\theta}=0$. From the $\mathrm{rHF}$ reference state, we create a broken symmetry state with two pairing orbitals as in Eq. (7), using two points at the FS, $\mathbf{k}$ (a highest occupied state) and $\mathbf{k}^{\prime}$ (a lowest unoccupied state), as illustrated in Fig. 4. The energy cost consists of kinetic and exchange terms [7]:

$$
\begin{gathered}
\Delta K \sim 2\left|c_{\mathbf{k}^{\prime}}\right|^{2} \frac{\hbar^{2}}{m} k_{F} \Delta k \\
\Delta V_{\mathrm{ex}} \sim\left|c_{\mathbf{k}^{\prime}}\right|^{2} \frac{e^{2}}{\pi} \Delta k \ln \frac{2 k_{F}}{\Delta k},
\end{gathered}
$$

where $k_{F}=1 /\left(\alpha r_{s}\right)$, with $\alpha=(4 / 9 \pi)^{1 / 3}$. Our choice of $\mathbf{k}$ and $\mathbf{k}^{\prime}$ gives: $\Delta k=\left|\mathbf{k}^{\prime}\right|-|\mathbf{k}| \sim\left(1 / 2 l_{F}\right)(2 \pi / L)=$ $1 /\left(2 l_{F}^{2} \alpha r_{s}\right)$, where $l_{F}$ is defined by $k_{F} \equiv l_{F}(2 \pi / L)$, i.e., $l_{F}=(3 / 8 \pi)^{1 / 3} N^{1 / 3}$. For fixed $r_{s}$, the exchange term dominates if $N$ is sufficiently large. Choosing $c_{\mathbf{k}}$ and $c_{\mathbf{k}^{\prime}}$ to be real and of $\mathcal{O}(1)$, we can write Eq. (9) as:

$$
\Delta V_{\mathrm{ex}}^{\mathbf{k k}^{\prime}} \sim \frac{e^{2}}{\pi \alpha r_{s}} \frac{\ln l_{F}}{l_{F}^{2}} .
$$

We now create a "satellite" pairing state in the vicinity of $\mathbf{k}$ and $\mathbf{k}^{\prime}$, i.e., with $\mathbf{k}^{\prime}-\mathbf{k}=\mathbf{s}^{\prime}-\mathbf{s}$ and $|\mathbf{s}-\mathbf{k}| \sim \mathcal{O}(2 \pi / L)$. We choose the excitation amplitude to be of the particular form: $c_{\mathbf{s}^{\prime}} \sim 1 /\left(\ln l_{F}\right)^{1+\delta}(\delta>0)$, and thereby $c_{\mathbf{s}} \sim 1$. Using Eq. (9) and noting that the difference in the magnitude of the wave vectors, $\Delta s \equiv\left|\mathbf{s}^{\prime}\right|-|\mathbf{s}|$, is less than the size of circles in Fig. [4 we obtain an upper bound to the energy cost for creating the $\left\{\mathbf{s}, \mathbf{s}^{\prime}\right\}$-pairing state

$$
\Delta V_{\mathrm{ex}}^{\mathbf{s s}^{\prime}} \sim \frac{2 e^{2}}{\pi \alpha r_{s}} \frac{1}{l_{F}\left(\ln l_{F}\right)^{1+2 \delta}} .
$$

The decrease in exchange energy because of "constructive interference" between the two parallel pairs is:

$$
\Delta V_{\mathrm{ex}}^{\mathrm{ks}} \sim-2 c_{\mathbf{k}} c_{\mathbf{k}^{\prime}} c_{\mathbf{s}} c_{\mathbf{s}^{\prime}} \frac{4 \pi e^{2}}{L^{3}} \frac{1}{|\mathbf{k}-\mathbf{s}|^{2}}
$$




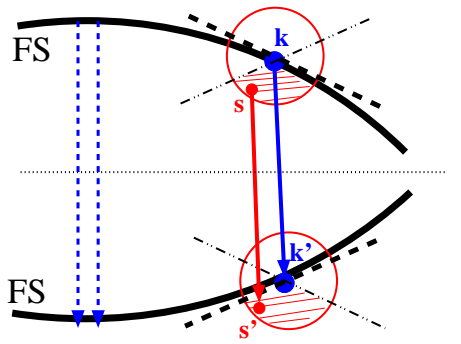

FIG. 4: (color online) Cartoon of pairing state with $q_{\sigma}<$ $2 k_{F}$. The primary pairing state $\left\{\mathbf{k}, \mathbf{k}^{\prime}\right\}$ is at the FS. The satellite pairing state $\left\{\mathbf{s}, \mathbf{s}^{\prime}\right\}$ can be in the shaded areas, within $\mathcal{O}(2 \pi / L)$ of $\mathbf{k}$ and $\mathbf{k}^{\prime}$. Dashed arrow lines illustrate Overhauser 3, 7] pairing at $2 k_{F}$.

$$
\sim-\frac{e^{2}}{2 \pi^{2} \alpha r_{s}} \frac{1}{l_{F}\left(\ln l_{F}\right)^{1+\delta}} .
$$

For sufficiently large $l_{F}$, i.e., a large enough system size, $\left|\Delta V_{\mathrm{ex}}^{\mathrm{ks}}\right|$ can always be made larger than the energy costs in Eqs. (10) and (11). Hence this is an SDW state with lower energy than the rHF state.

The wave vector of the constructed SDW is $\mathbf{q}_{\sigma}=\mathbf{k}^{\prime}-\mathbf{k}$. As Fig. 4 shows, $q_{\sigma}$ does not need to be $2 k_{F}$. The angle between $\mathbf{k}^{\prime}$ and $\mathbf{q}_{\sigma}, \theta$, can range from $0\left(q_{\sigma}=2 k_{F}\right)$ to $\pi / 2\left(q_{\sigma}=0\right)$. As $\theta$ increases, more $\left\{\mathbf{k}, \mathbf{k}^{\prime}\right\}$ pairing states become available on the FS, while the number of possible satellite pairs, i.e., the volume of the shaded areas, decreases. The optimal choice would be in between. In fact, as a crude estimate, the number of $\left\{\mathbf{k}, \mathbf{k}^{\prime}\right\}$ pairs is $\propto 2 \pi k_{F}^{2} \sin \theta$, and the number of $\left\{\mathbf{s}, \mathbf{s}^{\prime}\right\}$ pairs for each is $\propto(\pi-\theta)$. Maximizing their product gives $q_{\sigma} \sim 1.52 k_{F}$, which is consistent with our data.

In previous approaches [3, 7], pairing is constructed from orbitals directly across the FS. The energy lowering is driven by interference between such pairs (dashed arrows in Fig. 4), which in our model belong to primary pairing states. Our approach differs by including the satellite pairing states. The interference between the primary and satellite states is what makes a general $q_{\sigma}$ possible. This model is supported by the exact numerical data in Fig. 2. Clearly, the true ground state goes beyond this model: the energy will be further lowered by having more $\left\{\mathbf{s}, \mathbf{s}^{\prime}\right\}$ pairs and multiple $\left\{\mathbf{k}, \mathbf{k}^{\prime}\right\}$ states, etc.

We have shown that the uHF states at intermediate and high densities have nearly constant charge density. They are wave-like, and arise from pairing between states on the FS separated by distance $q_{\sigma}$. The momentum distribution is spin-independent. Its deviation from the Fermi sea is increasingly confined to the vicinity of the $\mathrm{FS}$ as $r_{s} \rightarrow 0$. The SDW wave vector is $q_{\sigma} \sim 1.5(2) k_{F}$, and its structures are determined primarily by the shortrange exchange potential [11]. In the rHF solution, the size of the exchange hole is $r_{x} \equiv 2.34 r_{s}$ [6]. A linear SDW with a wave length (i.e., characteristic like-spin separation) of $r_{x}$ has wave vector $q=1.40 k_{F}$. As $r_{s} \rightarrow 0$, the numerical results are sensitive to the detailed topology of the FS in the finite-size systems. The outcome of the SDW structure can vary greatly, as it is a delicate balance to optimize pairing among a small number of plane-wave states at the FS that can participate. Our results indicate that, at high densities, the HF ground state tends to further break spatial symmetry and favor one or two dimensions. As illustrated in the right panel of Fig. 3, for example, multiple 'pockets' can coalesce into locally or even globally (e.g., stripe-like) connected structures, in contrast with the Wigner crystal state where each pocket, corresponding to one electron, is fully localized.

To conclude, we have determined the true HF ground state for finite electron gas. Combining numerical and analytic results, we have described the origin and characteristics of the broken symmetry state at high density, and the novel pairing mechanism that drives it.

This work was supported by NSF (DMR-0535529 and DMR-0404853) and ARO (48752PH). We are grateful to H. Krakauer for help with the plane-wave machinery. We acknowledge useful discussions with H. Krakauer and R. M. Martin.

[1] E. P. Wigner, Phys. Rev. 46, 1002, (1934); Trans. Faraday Soc. 34678 (1938).

[2] F. Bloch, Z. Phys. 57, 549 (1929).

[3] A. W. Overhauser, Phys. Rev. Lett. 3, 414 (1959); Phys. Rev. 1281427 (1962).

[4] J. R. Trail et. al. Phys. Rev. B 68, 045107 (2003).

[5] D. Ceperley, Phys. Rev. B 18, 3126 (1978); D. M. Ceperley and B. J. Alder, Phys. Rev. Lett. 45, 566 (1980); F. H. Zong et. al., Phys. Rev. E 66, 036703 (2002).

[6] R. M. Martin, "Electronic Structure: basic theory and practical methods", Cambridge University Press, 2004.

[7] G. F. Giuliani and G. Vignale, "Quantum Theory of the Electon Liquid", Cambridge University Press, 2005.

[8] S. Zhang and H. Krakauer, Phys. Rev. Lett. 90, 136401 (2003); M. Suewattana et. al., Phys. Rev. B 75, 245123 (2007).

[9] C. Lin et. al., Phys. Rev. E 64, 016702 (2001).

[10] Systems with up to $N=66$ were projected to convergence following this approach. Some larger systems were quenched from a "frozen core" state, where $N_{\mathrm{fc}}(<N)$ electrons are frozen in the rHF state while the remaining $N-N_{\mathrm{fc}}$ electrons are active in the projection. $N_{\mathrm{fc}}$ is then gradually reduced in subsequent projections. This procedure can sometimes get "stuck" in a local minimum. It is thus possible that the energy gain in the larger systems is underestimated and is a lower bound.

[11] The structure which maximizes the near-neighbor distance (NND) between like-spin electrons is the closepacking fcc, which would lead to an NaCl-like SDW, but with a characteristic NND of $2.28 r_{s}$, less than $r_{x}$. 